\title{
A STEM-based Path Towards Atomic-scale Silicon-based Devices
}

Bethany M. Hudak ${ }^{1, *}$, Stephen Jesse ${ }^{1,2}$, Jiaming Song ${ }^{3, \#}$, Albina Borisevich $^{1}$, Paul C. Snijders ${ }^{3}$, Sergei V. Kalinin ${ }^{1,2}$, and Andrew R. Lupini ${ }^{1,2, *}$

1. Center for Nanophase Materials Sciences, Oak Ridge National Laboratory, Oak Ridge, TN, USA.

2. Institute for Functional Imaging of Materials, Oak Ridge National Laboratory, Oak Ridge, TN, USA.

3. Materials Science \& Technology Division, Oak Ridge National Laboratory, Oak Ridge, TN, USA.

\#. Present address: School of Physics, Northwest University, Xi' an, China

*Corresponding authors: hudakbm@ornl.gov, ar11000@ornl.gov

Precise control of material at the nanoscale is necessary for ever-shrinking devices that rely on quantum effects. The ubiquitous use of silicon in modern electronics makes it an ideal system for quantum-based nanoelectronic devices. However, direct patterning of silicon at the nanoscale is limited by current silicon growth and patterning processes such as lithography. The scanning transmission electron microscope (STEM) is a powerful tool for atomically resolved imaging and spectroscopy but recent work has demonstrated its utility as a platform for atom-by-atom fabrication [1-5]. The beam-matter interactions once thought of negatively as "beam damage" are now being harnessed to modify technologically relevant material at the nano- and atomic scales. STEM offers real-time imaging at room temperature, variable accelerating voltages for increased control over beam-matter interactions and can be paired with computer control and feedback for precise material manipulation. In addition to modifying materials using STEM, a hurdle remains for integrating a STEM sample into a functional device. Here, we discuss ways that we use the STEM to sculpt silicon and direct the placement of single dopants, as well as a potential route to bring these new techniques out of the microscope and into a functional device.

Two capabilities that can potentially postpone the end of Moore's empirical law are the ability to control semiconductor growth at the nm-scale and the ability to accurately position functional subsurface dopants. As shown in Figure 1a, we demonstrate the ability to sculpt silicon at the nanoscale using the atomically precise STEM electron beam [2]. We can crystallize, amorphize, and drill holes in silicon via control of the electron beam current. In addition to sculpting, we can reposition fronts of dopant atoms and position individual subsurface dopants (Figure 1b) [2,5].

In order to move these nanofabrication techniques from practice on a STEM sample, which is fabricated primarily for imaging, we must consider other methods to fashion samples that are artifact-free and sufficiently robust to withstand transfer from the STEM to a secondary substrate. Thin silicon membranes made by chemical etching of silicon-on-insulator (SOI) wafers, commonly used in CMOS devices, have been used for thin, flexible electronics and can be transferred between multiple substrates [6-8]. SOI wafers can be used to create transparent, silicon-based membranes suitable for STEM analysis. The silicon film on these wafers can be implanted with dopants (or otherwise modified) and then further adapted on the atomic-scale using the STEM. The robustness of these films provides a route to modify silicon in the STEM then transfer the film to a secondary substrate, enabling a potential route to transfer atom-by-atom fabrication in the microscope to a functional electronic device. [9]

\section{References:}

[1] S Jesse et al., Small 11 (2015), p. 5895. 
[2] S Jesse et al., Nanotechnology 29 (2019), p. 255303.

[3] T Susi et al., Ultramicroscopy 180 (2017), p. 163.

[4] O Dyck et al., Applied Physics Letters 111 (2017), p. 113104.

[5] BM Hudak et al., ACS Nano 12 (2018), p. 5873.

[6] GK Celler and S Cristoloveanu, Journal of Applied Physics 93 (2003), p. 4955.

[7] MM Roberts et al., Nature Materials 5 (2006), p. 388.

[8] SA Scott and MG Lagally, Journal of Physics D 40 (2007), p. R75

[9] Research supported by Oak Ridge National Laboratory's (ORNL's) Center for Nanophase Materials Sciences, which is a U.S. Department of Energy (DOE) Office of Science User Facility (SJ, SVK), ORNL's Laboratory Directed Research and Development Programs, managed by UT-Battelle, LLC for the U.S. DOE (BMH, JS, PCS), and the U.S. DOE, Office of Science, Basic Energy Sciences, Division of Materials Sciences and Engineering (AB, ARL). This manuscript has been authored by UT-Battelle, LLC, under contract DE-AC05-00OR22725 with the US Department of Energy (DOE). The US government retains and the publisher, by accepting the article for publication, acknowledges that the US government retains a nonexclusive, paid-up, irrevocable, worldwide license to publish or reproduce the published form of this manuscript, or allow others to do so, for US government purposes. DOE will provide public access to these results of federally sponsored research in accordance with the DOE Public Access Plan (http://energy.gov/downloads/doe-public-access-plan).
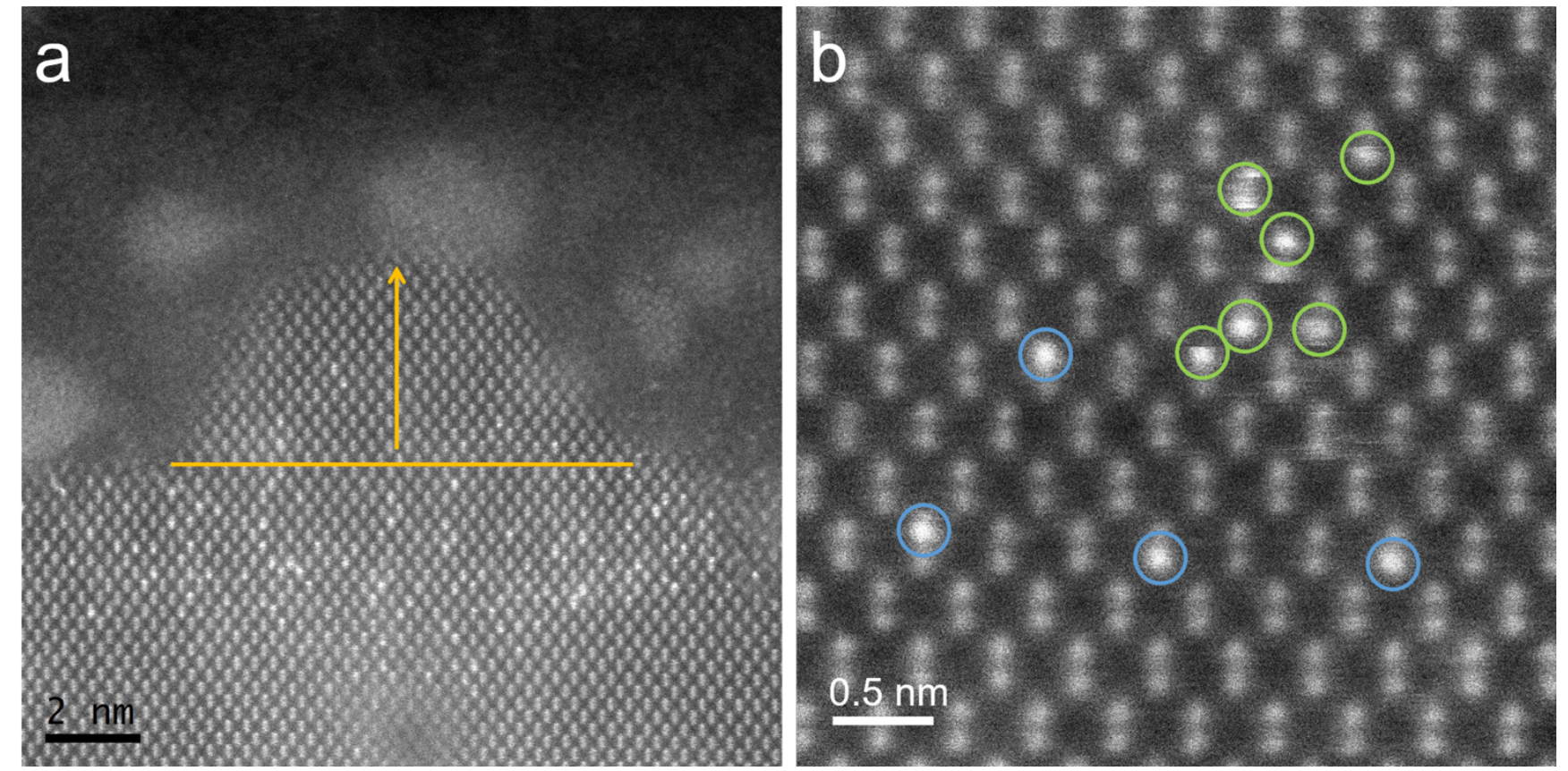

Figure 1. Using STEM for nano- and atomic-scale manipulation of matter. (a) Electron beam is scanned from a crystalline-amorphous silicon interface (horizontal orange line) into amorphous region (vertical direction of orange arrow), resulting in crystallized silicon. (b) Electron beam is used to directly position single bismuth dopants in silicon. Circles indicate bismuth atoms moved into region using electron beam. Green circles mark randomly dispersed dopants, blue circles indicate dopants that were deliberately repositioned. 